\title{
Blastomycosis Infection in The Setting of Immunosuppression with Concurrent Methotrexate and Adalimumab for Treatment of Psoriasis
}

\author{
Sebastian Otto-Meyer, BA ${ }^{1}$, Skylar Nahi, BA ${ }^{1}$, Ahmad Amin, MD ${ }^{1}$ \\ ${ }^{1}$ Department of Dermatology, Northwestern University Feinberg School of Medicine, Chicago, IL
}

\section{ABSTRACT}

Blastomycosis is a dimorphic fungi endemic to the Ohio/Mississippi Rivers and the Great Lakes that can infect immunocompetent and immunosuppressed individuals. Systemic fungal infections, such as histoplasmosis or blastomycosis, have often been reported in patients taking TNF- $\alpha$ inhibitors such as adalimumab. However, blastomycosis is significantly less common than histoplasmosis and no cases have been described in the literature. We report a case of systemic blastomycosis associated with adalimumab and methotrexate use for psoriasis and psoriatic arthritis treatment that required extensive treatment and discontinuation of both medications. To our knowledge, this is the first case of systemic blastomycosis associated with adalimumab and methotrexate treatment for psoriasis to be described in the literature.

\section{INTRODUCTION}

Blastomycosis is a dimorphic fungi that can infect both immunocompetent and immunocompromised individuals ${ }^{1}$. It is endemic to the Ohio/Mississippi Rivers area and the Great Lakes, where patients may inhale spores or fragments that transform to yeast in the lungs, resulting in pneumonia and flu-like symptoms, with occasional involvement of the skin and other organs. Endemic fungal infections, such as Histoplasmosis, Blastomycosis, and Coccidioidomycosis, have been frequently reported in patients taking TNF- $\alpha$ inhibitors, including adalimumab. Although sixteen cases of blastomycosis associated with TNF$-\alpha$ inhibitor use have been reported to the FDA adverse event reporting system, no systemic cases have been described with a detailed clinical course in the literature ${ }^{2}$. We present a case of pulmonary blastomycosis associated with adalimumab use in a patient with psoriasis.

\section{CASE REPORT}

A 71-year-old man with a history of psoriasis and psoriatic arthritis on adalimumab and methotrexate presented with two weeks of fatigue, decreased appetite, and night sweats. The patient's psoriasis had been well-managed on adalimumab for seven to eight years $(40 \mathrm{mg} / 0.8 \mathrm{ml}$ pen every other week) with a recent addition of $15 \mathrm{mg}$ methotrexate (MTX) weekly targeted at the psoriatic arthritis. Extensive work-up upon admission revealed enlarged perihilar and mediastinal lymph nodes on chest CT as well as positive blastomycosis and histoplasmosis urine antigens. The blastomycosis was considered to be the true positive, with 
histoplasmosis antigen positivity a likely cross-reaction. Diagnosis of disseminated blastomycoses was made. No skin findings concerning for blastomycosis were found.

Treatment was initiated with amphotericin B. However, rapidly worsening bilirubin and serum creatinine necessitated discontinuation. Oral itraconazole was started, but an elevation in liver enzymes resulted in a switch to voriconazole. As his symptoms resolved and labs normalized, he was discharged on a 12-month course of voriconazole. Adalimumab and methotrexate were discontinued upon initial admission and not restarted upon discharge. Outpatient bronchoscopy confirmed blastomycosis in pulmonary nodules, but the patient continued to do well on voriconazole. Dermatologic follow-up was unremarkable with no significant recurrence of psoriasis for 1.5 years. Mild psoriatic outbreaks were managed with topicals, including clobetasol $0.05 \%$ to body and hydrocortisone $2.5 \%$ to face. However, psoriasis worsened after 2 years off adalimumab despite consistent topical use, with extensive pruritic outbreaks on the legs, back, and trunk. At this point, a targeted IL-23 inhibitor (tildrakizumab-asmn) was started with improvement.

\section{DISCUSSION}

To our knowledge, this is the first descriptive case report of blastomycosis associated with adalimumab use published in the literature. Although sixteen cases are reported (including disseminated and cutaneous blastomycosis) within the FDA Adverse Event Reporting System (FAERS), the clinical details have not been established. Although methotrexate has been associated with blastomycosis in FAERS, the relatively small dose of methotrexate was unlikely to be the primary contributor. Here, we show that despite TNF- $\alpha$ inhibitor use along with methotrexate, the course of blastomycosis was relatively mild and able to be treated on an extended course of voriconazole. However, as 6 cases reported to the FDA resulted in death, this is clearly not always the case. We also demonstrate how his adalimumab was able to be safely stopped with minimal additional psoriasis for over a year, a fact that should be helpful guidance to dermatologists questioning the relevance of restarting a TNF- $\alpha$ inhibitor after infection.

This case raises several interesting concerns in the face of chronic TNF- $\alpha$ use. A metaanalysis of RCTs in patients with rheumatoid arthritis found that the use of biological DMARDs did not significantly increase the risk of invasive and superficial fungal infections (OR 1.31; 95\% Cl 0.46-3.72) or invasive fungal infections (OR 2.85; $95 \% \mathrm{Cl}$ 0.68-11.91).3 As such, a DMARD such as methotrexate (MTX) is less likely to be the sole etiology of the susceptibility to blastomycosis infection. Further, post licensure surveillance of adverse effects has associated the use of methotrexate concurrently with a TNF- $\alpha$ inhibitor (infliximab) with a higher risk for developing an endemic fungal infection of histoplasmosis ${ }^{4}$. In the literature today, adverse event studies and case series demonstrate an association between patients on a TNF- $\alpha$ inhibitor and MTX and increased susceptibility to dimorphic fungi infection. This case adds to the understanding of the these infections, demonstrating the details of an association between co-administration of a TNF- $\alpha$ inhibitor and MTX, with subsequent opportunistic infection by blastomycosis ${ }^{5}$.

This case report complements the incidence of histoplasmosis observed with TNF- $\alpha$ inhibitor therapy in the literature. It further supports the importance of screening all patients for risk factors prior to starting 
adalimumab and MTX therapy, and then annually. This includes questioning patients about travel to endemic regions and participation in activities that may increase risk of exposure ${ }^{6}$. Although there are no formal recommendations on screening strategies in high-risk patients, these patients may warrant closer monitoring. Indeed, this case illustrates that any patient experiencing concerning symptoms, such as prolonged fatigue, weight loss, or night sweats, should be carefully assessed if they are taking a TNF- $\alpha$ inhibitor. A consensus document on the safety of targeted and biological therapies demonstrated that no benefit is expected from the use of antibacterial, antiPneumocystis or antifungal prophylaxis in a patient cohort on anti-TNF-a therapy ${ }^{7}$. However, after infection, rigorous anti-fungal regimes are essential, and may be justified for extended periods.

\section{CONCLUSION}

In the case of the patient in this discussion, increase in baseline psoriatic activity may increase interest in restarting his adalimumab regimen. A history of a dimorphic fungal infection may justify consideration of fluconazole "maintenance." This can be recommended in those patients who desire to restart a TNF- $\alpha$ antagonist such as adalimumab, a recommendation guided by studies on fungal infection relapse in immunosuppressed patients who discontinued fungal prophylaxis ${ }^{8,9}$. If chronic maintenance therapy is not implemented, urine antigen screening at 3-month intervals may be performed to look for recurrence. Alternatively, as in this case, a new biologic, such as an IL-17 or IL-23 inhibitor, can be started. While these also may impair antifungal immunologic defenses, a recent review found no reports of invasive fungal disease following treatment of psoriasis or other inflammatory conditions with $\mathrm{IL}-17$ or IL-23 inhibitors ${ }^{10}$.

\section{Conflict of Interest Disclosures: None}

Funding: None

Corresponding Author:

Ahmad Amin, MD

Department of Dermatology

Feinberg School of Medicine

111 W Washington St Ste 1801

Chicago, IL 60602

Email: ahmad.amin@nm.org

\section{References:}

1. McBride JA, Gauthier GM, Klein BS. Clinical Manifestations and Treatment of Blastomycosis. Clin Chest Med. 2017;38(3):435-449.

2. FDA Adverse Events Reporting System (FAERS) Public Dashboard. Accessed January 7, 2021.

3. Kourbeti IS, Ziakas PD, Mylonakis E. Biologic Therapies in Rheumatoid Arthritis and the Risk of Opportunistic Infections: A Meta-analysis. Clinical Infectious Diseases. 2014;58(12):16491657.

4. Lee JH, Slifman NR, Gershon SK, et al. Lifethreatening histoplasmosis complicating immunotherapy with tumor necrosis factor alpha antagonists infliximab and etanercept. Arthritis Rheum. 2002;46(10):2565-2570.

5. Crum NF, Lederman ER, Wallace MR. Infections Associated With Tumor Necrosis Factor- $\alpha$ Antagonists. Medicine. 2005;84(5):291302.

6. Toussi SS, Pan N, Walters HM, Walsh TJ. Infections in children and adolescents with juvenile idiopathic arthritis and inflammatory bowel disease treated with tumor necrosis factor- $\alpha$ inhibitors: systematic review of the literature. Clin Infect Dis. 2013;57(9):1318-1330.

7. Baddley JW, Cantini F, Goletti D, et al. ESCMID Study Group for Infections in Compromised Hosts (ESGICH) Consensus Document on the safety of targeted and biological therapies: an infectious diseases perspective (Soluble immune effector molecules [I]: anti-tumor necrosis factor- $\alpha$ agents). Clin Microbiol Infect. 2018;24 Suppl 2:S10-s20.

8. Mathew G, Smedema M, Wheat LJ, Goldman M. Relapse of coccidioidomycosis despite immune reconstitution after fluconazole 
secondary prophylaxis in a patient with AIDS.

Mycoses. 2003;46(1-2):42-44.

9. Wood KL, Hage CA, Knox KS, et al.

Histoplasmosis after treatment with anti-tumor necrosis factor-alpha therapy. Am J Respir Crit Care Med. 2003;167(9):1279-1282.

10. Lee MP, Wu KK, Lee EB, Wu JJ. Risk for deep fungal infections during IL-17 and IL-23 inhibitor therapy for psoriasis. Cutis. 2020;106(4):199-

205. 